Document downloaded from:

http://hdl.handle.net/10251/170275

This paper must be cited as:

Selim, H.; Delgado-Prieto, M.; Trull, J.; Picó Vila, R.; Romeral, L.; Cojocaru, C. (2020). Defect reconstruction by non-destructive testing with laser induced ultrasonic detection. Ultrasonics. 101:1-8. https://doi.org/10.1016/j.ultras.2019.106000

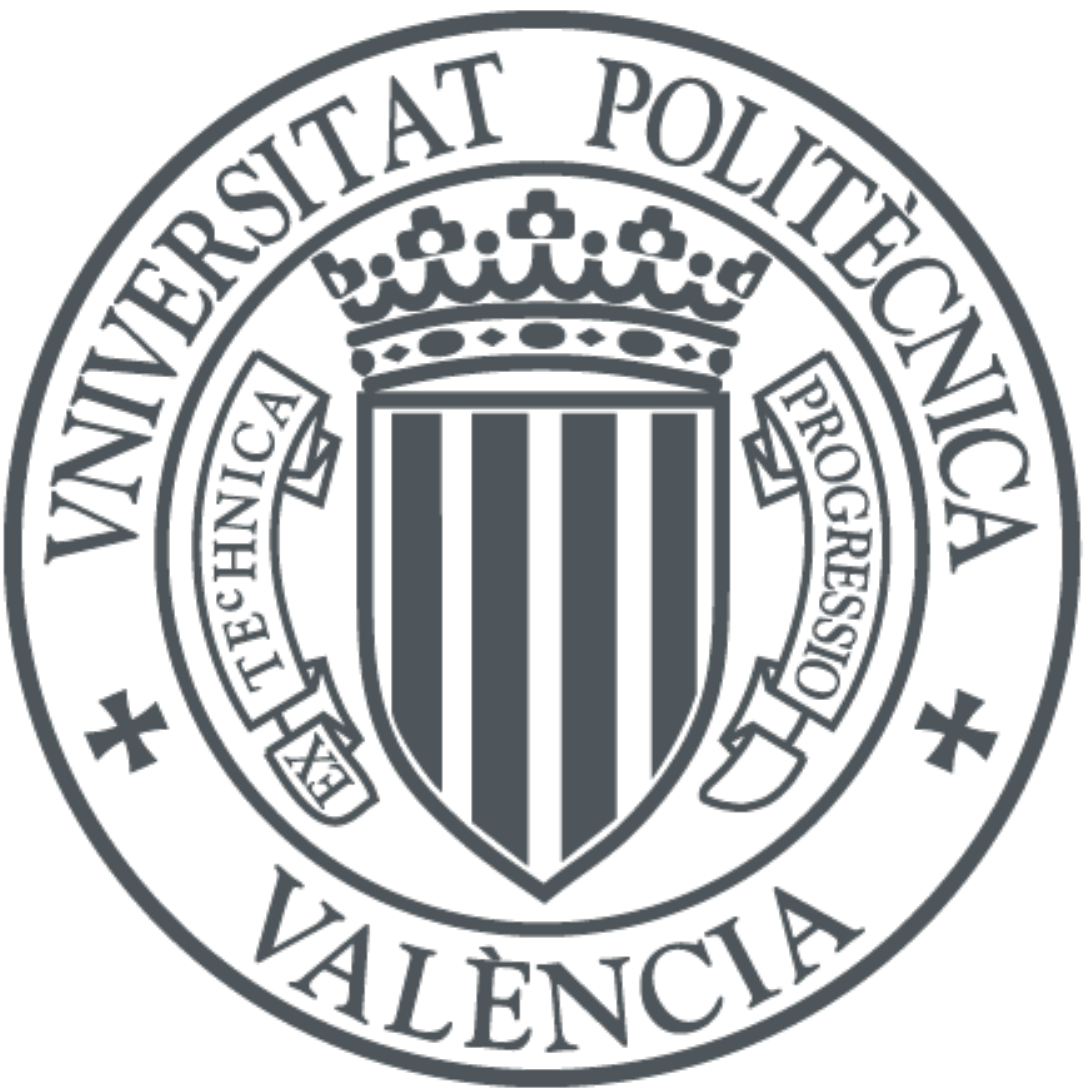

The final publication is available at

https://doi.org/10.1016/j.ultras.2019.106000

Copyright Elsevier

Additional Information 


\title{
Defect reconstruction by non-destructive testing with laser induced ultrasonic detection
}

\author{
Hossam Selim $^{\mathrm{a}, *}$, Miguel Delgado-Prieto ${ }^{\mathrm{b}}$, Jose Trull ${ }^{\mathrm{a}}$, Rubén Picóc ${ }^{\mathrm{c}}$, Luís Romeral ${ }^{\mathrm{b}}$, Crina \\ Cojocaru $^{\mathrm{a}}$ \\ ${ }^{a}$ Physics Department, Universitat Politècnica de Catalunya, Terrassa, Barcelona, Spain \\ ${ }^{b}$ Electronic Engineering Department, Universitat Politècnica de Catalunya, Terrassa, Barcelona, Spain \\ ${ }^{c}$ Instituto de Investigación para la Gestión Integrada de Zonas Costeras, Universitat Politècnica de \\ Valencia, Valencia, Spain
}

\begin{abstract}
This work envisages a detailed study of two-dimensional defect localization and reconstruction, using laser generated ultrasound and its application as a remotely controlled nondestructive testing method. As an alternative to full ultrasonic or full optical approaches, we propose a hybrid configuration where ultrasound is generated by impact of laser pulses, while the detection is done with conventional transducers. We implement this approach for defect reconstruction in metallic elements and show that it combines advantages of both photonic and ultrasonic devices, reducing the drawbacks of both methods. We combine our experimental results with a high-resolution signal processing procedure based on the synthetic aperture focusing technique for the benefit of the final two-dimensional visualization of the defects.
\end{abstract}

Keywords: laser ultrasonics, defect reconstruction, non-destructive testing, synthetic aperture focusing technique, NDT, SAFT, B-scan

\section{Introduction}

Non-Destructive Testing (NDT) methods are nowadays implemented in industrial applications for the detection of fractures and defects in different materials [1]. The most extended NDT techniques involve transducers generating ultrasound waves and detecting them after the propagation through the material [2]. Typically, they excite ultrasound using conventional transducers placed in contact with the sample and they analyze the ultrasonic pulse's Time Of Flight (TOF) through the material under test from the transducer to the receiver in order to identify discontinuities in the wave propagation. This approach was extensively discussed in many research papers where defects were localized in space, e.g. the measurement of the crack location, height and width [3][4][5], or of the crack penetration, using ultrasonic guided waves [6]. The detection commonly relies on pulse-echo or

\footnotetext{
*Corresponding author.

Email address: hossam.eldin.mohamed.selim@upc.edu (Hossam Selim)
} 
pitch-catch modes. Pulse-echo mode uses the same ultrasonic transducer to generate and detect the signals and for a good detection, the defect should be vertically aligned with the sensor. The pitch-catch mode uses an emitter and a receiver and has more flexibility to work both in transmission and reflection modes. This technique can take measurements at different angles, but it is more expensive as it requires many sensors and more complex data processing [7][8]. Ultrasonic signal frequency, ranging from fractions of $\mathrm{MHz}$ up to $20 \mathrm{MHz}$, affects the sensitivity and resolution of the measurement. At higher frequencies, smaller defects can be detected more accurately, but also surface scattering reduces the penetration depth. These methods have the advantages of low cost, easy implementation and provide satisfactory results in many applications. Among the drawbacks we mention low output power preventing such systems to be used remotely, low frequency bandwidth range that makes necessary the use of transducer arrays, small excitation areas that prevent covering large object areas at once and require ultrasonic scanners, and quite low spatial resolution in the excited volume and in the detection.

As an alternative, all-optical methods known as laser-ultrasonics, based on laser generated ultrasound waves and optical detection of the propagated waves, showed up in the NDT field, offering the possibility of remote excitation and detection at a much higher resolution [9][10].The laser pulse is rapidly absorbed into a shallow volume of the material inducing a stress wave that generates an acoustic pulse [10]. The detection of the transmitted and/or reflected signals can be done as well using optical systems detecting the vibration created by the acoustic wave at the surface, as for example optical interference [11] or techniques of holographic interferometry [9]. All-optical methods have important advantages such as the remote non-contact application and control, the generation of broadband frequency wave spectrum (from $\mathrm{KHz}$ to $\mathrm{GHz}$ ), high output power and the possibility to easily scan a larger object area at once. As a drawback we mention the critical mechanical stability and the need for an anti-vibration setup in order to obtain reliable results, which makes them difficult to apply in certain fields.

In this work, we propose a hybrid system that combines the advantages of the optical systems for remotely laser-generated ultrasonic with conventional transducers for detection. The ultrasound will be induced by laser pulses, as in the all-optical methods, allowing inspection from a far distance from the object and enabling a scan of the test specimen remotely, without the need of a direct contact. Laser generated ultrasound also provides a broad frequency bandwidth excitation compared with the limited bandwidth of ultrasonic transducers, covering the whole ultrasonic bandwidth needed for different applications. On the other side, the use of traditional transducers (contact or contactless) for the ultrasound detection removes the interferometric stability problems in the all-optical techniques. The hybrid system allows an improved measurement of the defects location and size by implementing scan measurements over a specific surface area. We combine our experimental results with a signal processing technique based on synthetic aperture focusing technique (SAFT). SAFT technique is based on the principle of superposition of multiple signals captured at different positions on the surface of the object under test and shifted numerically in time by a delay corresponding to the spatial displacement of the exciters/receivers. This generates a focused image of the defect out of multiple unfocused images. This focused image would 
have a much higher amplitude at the defect position due to the diffraction of the waves at the defect boundaries compared to healthy positions inside the object of interest. SAFT has the advantage of being able to visualize the whole volume providing $2 \mathrm{D}$ or $3 \mathrm{D}$ information about the object depending on the resolution and the number of scans performed on the object [12][13][14][15]. Our contribution mainly considers visualizing the defect using SAFT algorithm in volumetric regime instead of two dimensional regimes (depth plane) we found in the literature. This is achieved through a hybrid system using Laser as an exciter and a contact transducer as a receiver. To our knowledge, the articles discussing SAFT algorithm were mainly interested in scanning the receivers across the scan line/area or scanning both transmitter and receivers. However, in our case we are avoiding scanning the receivers and we are interested to scan the exciters with a lot of scan points with high step resolution, thanks to the Laser source that make it much faster, easier and reliable.

\section{Experimental set-up}

The set-up designed for our experiments is shown schematically in Figure (1). We use an Nd:YAG laser doubled in frequency, emitting pulses of $8 \mathrm{~ns}$ at a wavelength of $532 \mathrm{~nm}$, with an energy per pulse of $10 \mathrm{~mJ}$. The focused laser beam impacts onto the surface of the object under study where the pulse is rapidly absorbed into a shallow volume of the material and creates a localized thermo-elastic expansion. This expansion induces a stress wave and an acoustic pulse generating broadband ultrasound waves that propagate inside the material. The laser beam can scan a selected area of the object surface by means of a programmable XY galvanometer. For each excitation point, the ultrasonic waves propagate through the object, they are reflected or scattered by different material particles in the volume, and then is detected by the ultrasonic transducer.

For the detection we use two conventional ultrasonic sensor transducers (Olympus V133$\mathrm{RM}$ ) at $2.2 \mathrm{MHz}$ central frequency, coupled to the surface of the object. The sensors can be placed on the incident surface (measuring reflection/scattering) as well as on the opposite side (measuring transmission). The signal collected by the sensors is sent to a preamplifier (Olympus 5662), connected to a high-performance Gage A/D card (50 MHz sampling frequency, 16 bit of resolution), linked to a computer for further data processing. For each excitation point the transducer records a voltage/time (A-scan) data set.

We analyze two aluminum cuboid samples with identical dimensions of $300 \mathrm{~mm} \times 200$ $\mathrm{mm}$ x $20 \mathrm{~mm}$. One sample is homogeneous, without any holes or defects, referred in the following discussion as "healthy". The second one, shown in Figure (2), has a cylindrical hole with a diameter of $8 \mathrm{~mm}$ and hole depth of $85 \mathrm{~mm}$ and is referred to from now on as "unhealthy" sample. Two different experiments were performed on this sample: (i) the laser performs a 1D scan (B-scan) and the detection is made with only one transducer; (ii) the laser perform a 2D scan, we use two transducers for detection and we apply SAFT algorithm for the signal processing. The laser scan is performed on the $300 \mathrm{~mm}$ x $200 \mathrm{~mm}$ face of the cuboid and the receiving transducers are located at the same face of the cuboid where laser excitation is performed, i.e; working in reflection mode. Figure (2) shows the laser scanning line/area and the sensors positions in each experiment. 


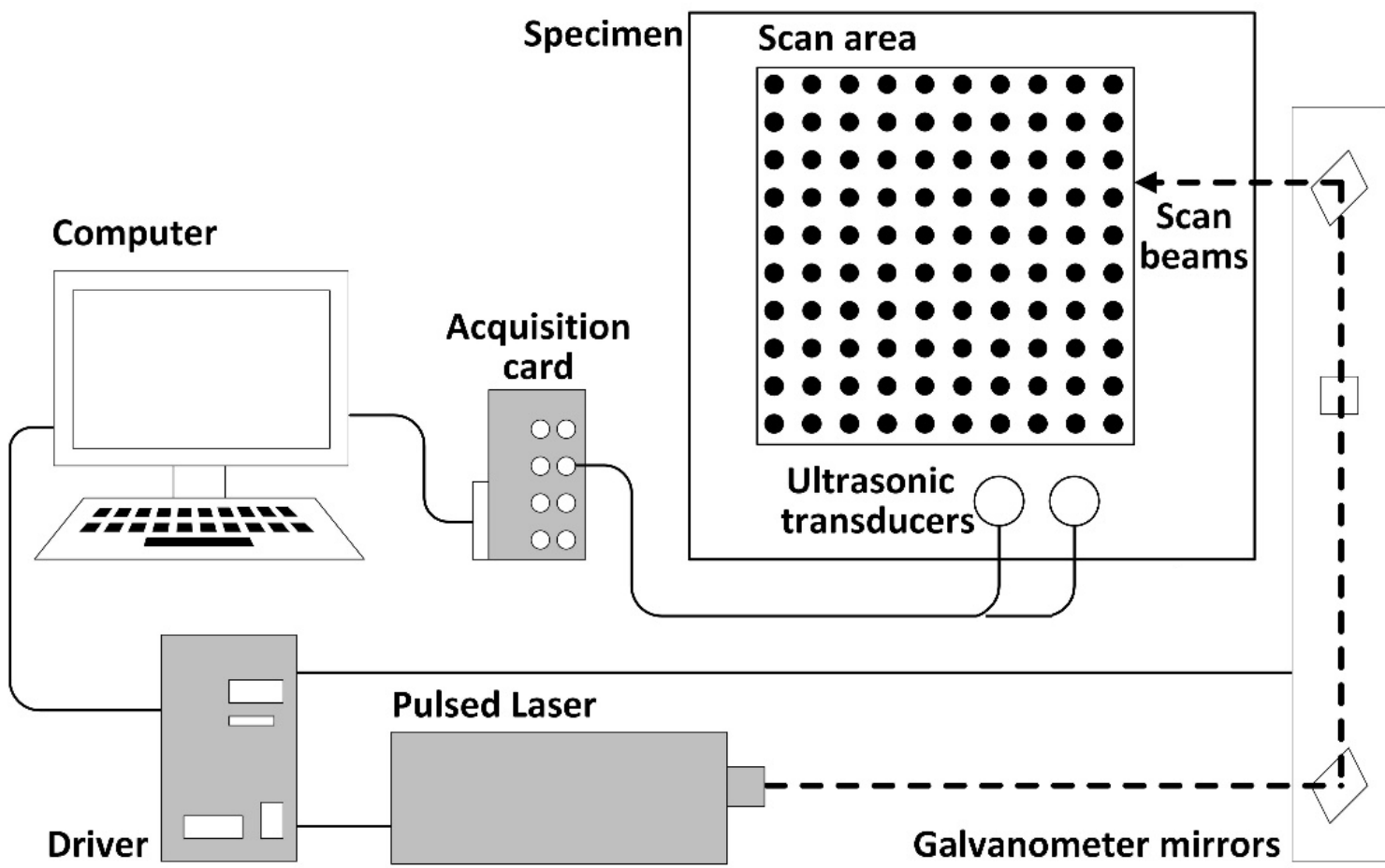

Figure 1: Schematic representation of the experimental set-up

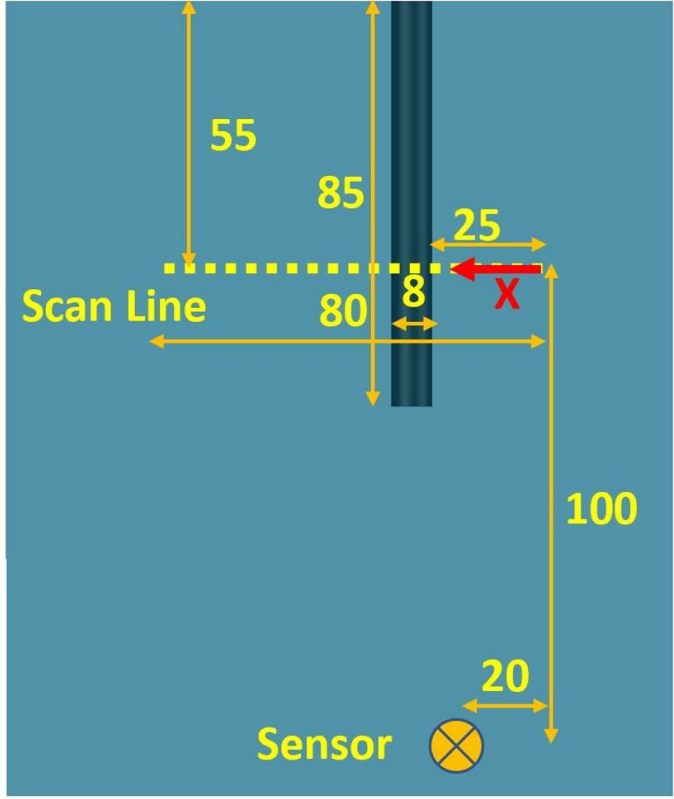

a)

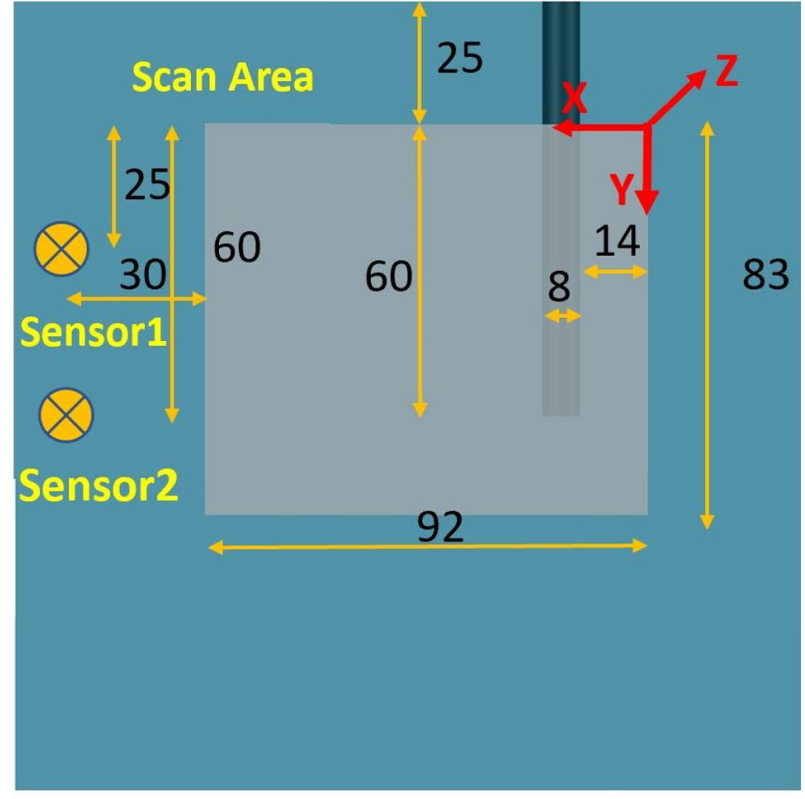

b)

Figure 2: Experimental sample with a) scan line and ultrasonic sensor positions for B-scan experiment and b) scan plane in grey for SAFT experiment and ultrasonic sensors for second experiment. 
a)

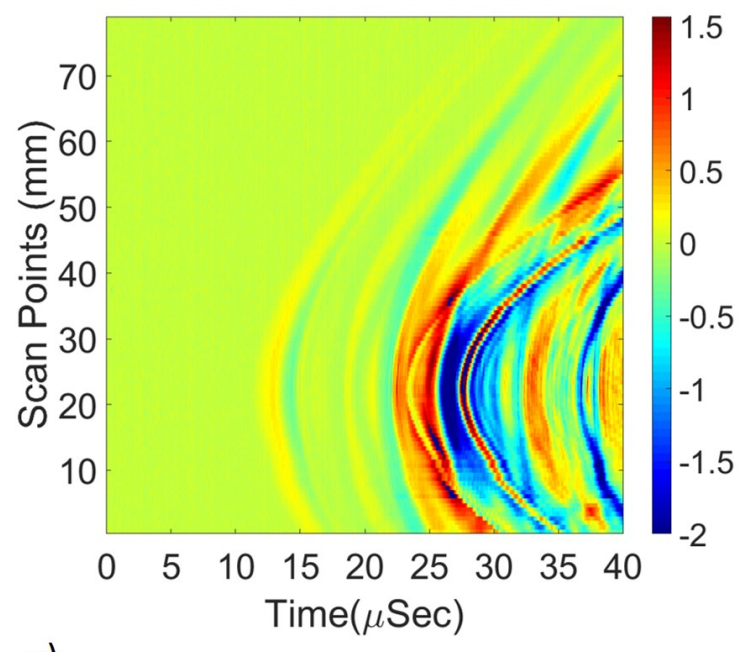

Figure 3: a) B-scan results of the healthy b) B-scan results of the unhealthy sample and an inset with zooming the discontinutites in the result.

\section{B-scan and signal processing}

In the first experiment the galvanometer was programmed to execute a 1D scan (Bscan) along one line of the object surface in the horizontal (X) direction, crossing on top of the defect, Figure (2a). We selected 120 scan points covering a distance of $80 \mathrm{~mm}$. For each excitation point the ultrasound waves detected by the sensor were recorded using our data acquisition system. For the signal processing we applied a band pass filter of $3 \mathrm{MHz}$ bandwidth around the $2.25 \mathrm{MHz}$ central frequency and an interpolation algorithm to reduce the excessive oscillation noise and unnecessary perturbations.

The B-scan results for all processed signals are shown in Figure (3). For the healthy sample in Figure (3a) and for the unhealthy one in Figure (3b). We plot the position of the scan points as a function of the time of flight (TOF) frames, while the color bar represents the intensity of the signal. A higher intensity is a sign of a high reflection towards the sensor coming from a reflecting source that can be a boundary or a defect embedded in the object under test.

The TOF of the waves depends on the distance between the laser incidence point and the acoustic sensor's position. The first wavefront arriving from the laser to the sensor starts to appear at $\mathrm{t}=11 \mu \mathrm{s}$ for both samples, since the relative position of the laser scan line and the sensor was the same. For the healthy sample there is no visible perturbation in the B-scan map, due to the homogeneity of the sample. All reflections come from the borders. For 
the unhealthy sample we see the same boundary reflections, overlapped with the reflections coming from the defect. The latter ones alter the B-scan map, showing a major discontinuity and perturbations that correspond to the effect of the hole. As highlighted in Figure (3b), the discontinuity clearly appears in the range between $\mathrm{X}_{1}=25 \mathrm{~mm}$ and $\mathrm{X}_{2}=36 \mathrm{~mm}$, respectively $(\Delta \mathrm{X}=11 \mathrm{~mm})$, on the vertical axis and it is centered at scan position $\mathrm{X}=30.5 \mathrm{~mm}$.

In Figure 3b, we have added an inset inside the figure for better visualization of these discontinuities with reduced limits of the color map to exaggerate the effect of the discontinuities and the arrows are indicating the points of discontinuity. We need to emphasize that the echoes from the hole are reflected from both the front and rear surfaces of the cylinder. Front surface is providing higher intensity due to this reflection, while the signal passing to the rear surface is already moving in the air in the hole until it reaches the rear surface and then reflected back to the transducer (passing again by the air in the hole). This attenuates the signal and causing lower amplitude for this reflection. Other echoes appearing later in time axis are multiple reflections from defect and boundaries and include a lot of interference, so they are not essential in this analysis. These main reflections are highlighted by the arrows in the larger Figure $3 \mathrm{~b}$ with the full color map range.

These values are very close to the actual hole diameter of $8 \mathrm{~mm}$ and its position on the sample, with a sizing error of $37 \%$ (the defect appears to be bigger than it really is), and a positioning error of $0 \%$.

This first experiment shows that B-scan technique, using a remote 1D laser scan and only one sensor, is a very simple and robust technique that provides reliable information about general position of defect location in a fast process. The resolution of the results is comparable with the best commercial NDT full ultrasonic devices but have the advantages of noncontact excitation and remote control of the scan. However, this method does not allow a $2 \mathrm{D}$ reconstruction of the defect.

\section{2D scan and SAFT analysis}

When a 2D or 3D reconstruction of the defect is envisaged, a 2D scan configuration, easily achievable with our laser induced excitation system, is required. We programed our experimental setup, shown in Figure (1), to perform a 2D scan over a MxN matrix points by remotely controlling the position of laser incident point onto the object surface. The scanning area (in grey) as well as the position of the receivers (in yellow) in this second experiment are shown in Figure $(2 \mathrm{~b})$. For each excitation point, each receiver records a corresponding A-scan signal. The experimental results combined with the SAFT technique are expected to give accurate information about the area of the defect for getting a precise $2 \mathrm{D}$ visualization of its location and dimensions.

SAFT is commonly used for the signal processing in the characterization of embedded defects in the volumetric regime. The technique is based on the delay and sum (DAS) principle, that superimposes the relatively low-resolution A-scan data at every excitation point of the scanned area, creating a higher resolution focused image, with a higher signal to noise ratio (SNR), referred to as the synthetic aperture (SA). Depending on the implemented setup, the analysis can be performed in the time or frequency domain [12][13][14]. SAFT 
can generate a 2D image of a particular plane of the object, or can be generalized to the $3 \mathrm{D}$ mode to cover all planes in a volume. The reconstructed 3D image can project maps of the defect in any image plane, regardless of the plane used for the scanning of the ultrasonic signals. In the time domain analysis, the TOF is calculated across all SA points, starting from the excitation signal source position at a particular scan index to all geometrical target points in the object's volume and back to the signal receiver, for each particular scan point. For each geometrical point, an integration of all received signals at a particular value of TOF is calculated, to produce the final pixel value of this particular scan point in the final image. This integration results in a higher contrast, resolution and better SNR value[14][15]. Figure (4) shows a schematic representation of the SAFT technique, assuming a 3D object under investigation with an arbitrary scatter point $(\mathrm{P})$ and an exciter-receiver set in a pitch-catch mode, similar to our experimental set-up. We consider an arbitrary excitation point (T) generating spherical waves and a receiver $(\mathrm{R})$, both placed on the XY plane. The SA top view is assumed to be on the XY plane.

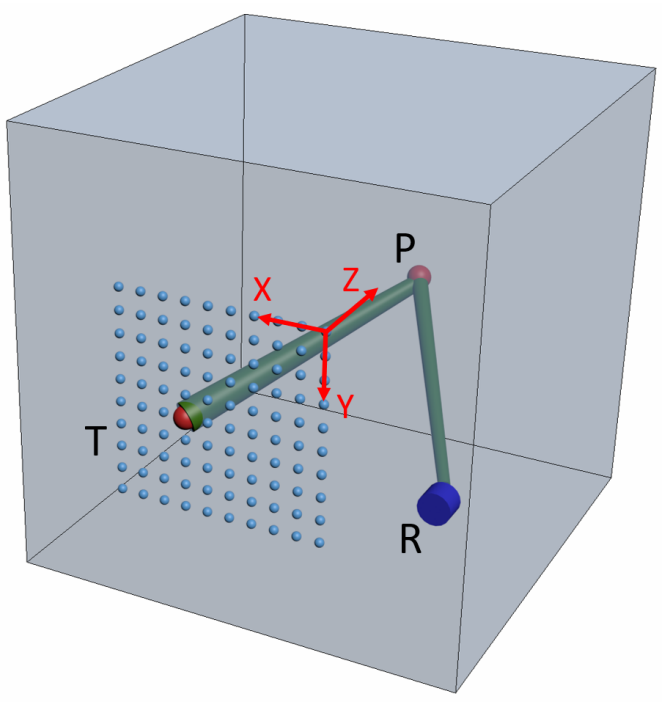

Figure 4: Scheme of the SAFT technique processing used for the object detection. A wave is sent from a point of interest $(\mathrm{P})$ by a transmitter $(\mathrm{T})$. It is reflected/scattered by the defect and measured at the receiver $(\mathrm{R})$. The procedure is repeated with the transmitter in every point of interest.

The $\operatorname{TOF}_{(i, j, k) P}$ of the ultrasonic signal generated by $\mathrm{T}$ and detected by $\mathrm{R}$ after it has been propagated to and reflected by the arbitrary point $(\mathrm{P})$, can be calculated as:

$$
\operatorname{TOF}_{(i, j, k)_{P}}=\frac{\left|\vec{d}_{(i, j, k)_{P}}-\vec{d}_{(i, j, 0)_{T}}\right|+\left|\vec{d}_{(i, j, k)_{P}}-\vec{d}_{(i, j, 0)_{R}}\right|}{c}
$$

where $d$ is the displacement vector at $\mathrm{T}, \mathrm{R}$ and $\mathrm{P}$ positions, $i, j, k$ are the indexes of the volume image points in the $\mathrm{X}, \mathrm{Y}, \mathrm{Z}$ planes respectively, and $c$ denotes the speed of the longitudinal waves in the material [12][16][17][18]. This calculation is done for every point in the A-scan measurements to produce a preliminary unfocused image $\mathrm{y}_{R}$ of this particular 
point $\mathrm{P}$. The combination of all individual images results into a high resolution focused image $\mathrm{y}_{f}$. We assume that the scan is done for $\mathrm{MxN}$ points denoting the number of the $2 \mathrm{D}$ excitation steps over the SA, and that only one receiver collects all the transmitted A-scan data from all scan points at an arbitrary point in the SA. The final signal is given by:

$$
y_{f}\left(\vec{R}_{P}\right)=\sum_{j=1}^{N} \sum_{i=1}^{M} y_{R}\left(\operatorname{TOF}_{(i, j, k)_{P}}, i, j\right)
$$

Where $\mathrm{y}_{R}$ is the measured A-scan signal amplitude at the corresponding $\operatorname{TOF}_{(i, j, k) P}$ at scan point $i$ and $j$ and $\mathrm{y}_{f}$ is the focused signal received from all the scan points at this particular receiver $\mathrm{R}$. This can be regarded as an integration of all received signals from $\mathrm{MxN}$ excitation points at the SA.

In equation (2) we need to add a coefficient that represents the effect of the attenuation and decay in the acquired signal when it passes from the exciter to the scattered point and back to the receiver. This coefficient is called weighting function or apodization function: $\mathrm{a}\left(\operatorname{TOF}_{(i, j, k) P,}, \mathrm{i}, \mathrm{j}\right)[16]$.

$$
y_{f}\left(\vec{R}_{P}\right)=\sum_{j=1}^{N} \sum_{i=1}^{M} a\left(T O F_{(i, j, k) P}, i, j\right) * y_{R}\left(\operatorname{TOF}_{(i, j, k) P}, i, j\right)
$$

In order to account for the diffraction in propagation, the apodization function applies lower weights to the signals captured at the far ends of SA and filters the unfocused signals in the SAFT analysis. Equation (3) represents the DAS where summation is applied to the delayed versions of the signals at the corresponding scan points.

The focused signal results from the superposition of the A-scan data from MxN excitation points captured by one receiver, where each A-scan itself is a superposition of signals coming from point scatters in the whole volume. If we go further and we assume that we could implement also a $\mathrm{MxN}$ scanning matrix for the receiver position, equation (3) can be modified to consider the integration of all receivers as well. In this case each image point will be constructed from $(\mathrm{MxN})^{2}$ points of integration instead of $\mathrm{MxN}$ scan points as for the case when we use only one receiver. SAFT can, thus, be applied to any image of all planes to produce an image for the defect from any projection direction.

There are several assumptions that we have to make when we apply SAFT to the final image reconstruction. The exciter is considered to be a point source, which perfectly applies for the laser generated ultrasound. Receivers are assumed to be point-like transducer: the smaller the size, the less numerical errors in the algorithm. The laser scanning area and the receiver locations are assumed to be far enough from the object's boundaries to avoid reflections that could cause misleading data. The medium is assumed to be homogeneous, with non-spatial dependent physical parameters. In other scenarios where the wave dispersion could be significant due to the presence of composite or inhomogeneous materials, the SAFT algorithm should be modified to account for different wave velocities and wave diffraction.

Experimental measurements have been conducted in a pitch-catch mode, using two receivers placed at the fixed positions indicated in Figure $(2 \mathrm{~b})$. The laser has been programmed to scan an area of $92 \mathrm{~mm} \times 83 \mathrm{~mm}$, with 101 x 91 scan points. The signals captured by the two sensors have been recorded using the amplifier and data acquisition system described 
in section 3, and were pre-processed using a band pass filter and an interpolation algorithm to remove the background noise and low/high frequency components. Then, an averaging algorithm was applied to remove the DC components from the signal. The SAFT algorithm was finally applied, considering a Gaussian weighting function.

The reason we did not add more receivers (e.g: microphone array) is that our main focus is to use the transmitter, not the receiver, as the scanning mechanism with a lot of points with high step resolution. With laser excitation, we can do the scanning of the transmitter with a step size of $0.9 \mathrm{~mm}$ for more than 9000 scan points. This is very difficult to achieve with conventional arrays. In addition, the scanning with the laser makes the incident pulse on the object surface equivalent to a point source, which is better for the SAFT efficiency.

As a result, we obtain the volume reconstruction of the selected scanned area shown in Figure (5a), where the defect corresponds to the yellow shadows. The position and dimensions of the defect can be better visualized and measured if we select projections of different cross-sections in the volume of interest, that can be extracted from Figure (5) for any required coordinates. Figures (5b) and (5c) show two cross-section slices in the XY and $\mathrm{XZ}$ planes respectively. From these projections we measure a defect with horizontal $(\Delta \mathrm{X})$ size of $8.2 \mathrm{~mm}$ and vertical $(\Delta \mathrm{Y})$ size of $56 \mathrm{~mm}$.

The contour map of one of the cross-sections is another representation of the results that can even better visualize the defect. Figure (6) shows the contour map of the XY cross-section, enabling the reconstruction of the $\mathrm{X}$ and $\mathrm{Y}$ dimensions: the defect is centered at $\mathrm{X}=20 \mathrm{~mm}$ and $\mathrm{Y}=40 \mathrm{~mm}$ with the corresponding size $\Delta \mathrm{X}=8.2 \mathrm{~mm}$ and $\Delta \mathrm{Y}=56 \mathrm{~mm}$. Compared with the real size and position of the defect in our sample, the resulting reconstruction has the following errors: a sizing error of $4.6 \%$, concretely, $\Delta \mathrm{X}_{\text {error }}=2.5 \%$ and $\Delta \mathrm{Y}_{\text {error }}=6.7 \%$ and a positioning error of $12.25 \%$, concretely, $\mathrm{X}_{\text {error }}=2.5 \%$ and $\mathrm{Y}_{\text {error }}=22 \%$. There are several reflections in the resulting images that do not come from the defect, that could be misleading. The contour map helped to identify and ignore them. These reflections can be overcome in further experiments.

The reflections appearing in the reconstructed images, as well as the resolution of the reconstruction are conditioned by some aspects of our experiment. On one hand, Z-dimension of the object studied here was too small compared with $\mathrm{X}$ and $\mathrm{Y}$ ones. In other words, the distance between the laser/sensor and defect is larger than the one to the $\mathrm{Z}$ boundaries of the object itself. This results in multiple reflections which arrive to the receiver faster than those coming from the defect, leading to misleading wavefronts that produce some errors in the reconstructed results. To solve this problem, $\mathrm{Z}$ dimension of the object under test should be large enough to avoid internal reflections from the boundaries. This applies to all object boundaries in all directions. On the other hand, in our experiment we fixed the position of the receiving sensors and only scanned the laser impact points. This change in laser scan point position results in a change in the angle between the excitation point and the ultrasonic receiving sensor. This angle at certain points of scan increases significantly due to object geometry. Large angles reduce the performance of the SAFT technique and can significantly reduce the signal-to-noise ratio. These constraints can be resolved by increasing the object's depth and reducing the angle by scanning the receivers along with the laser beam. 


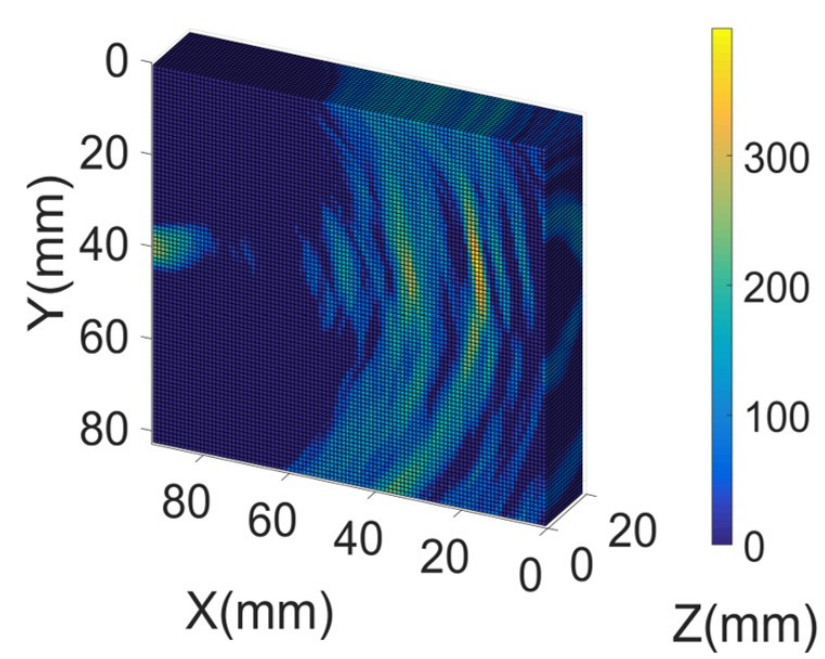

a)

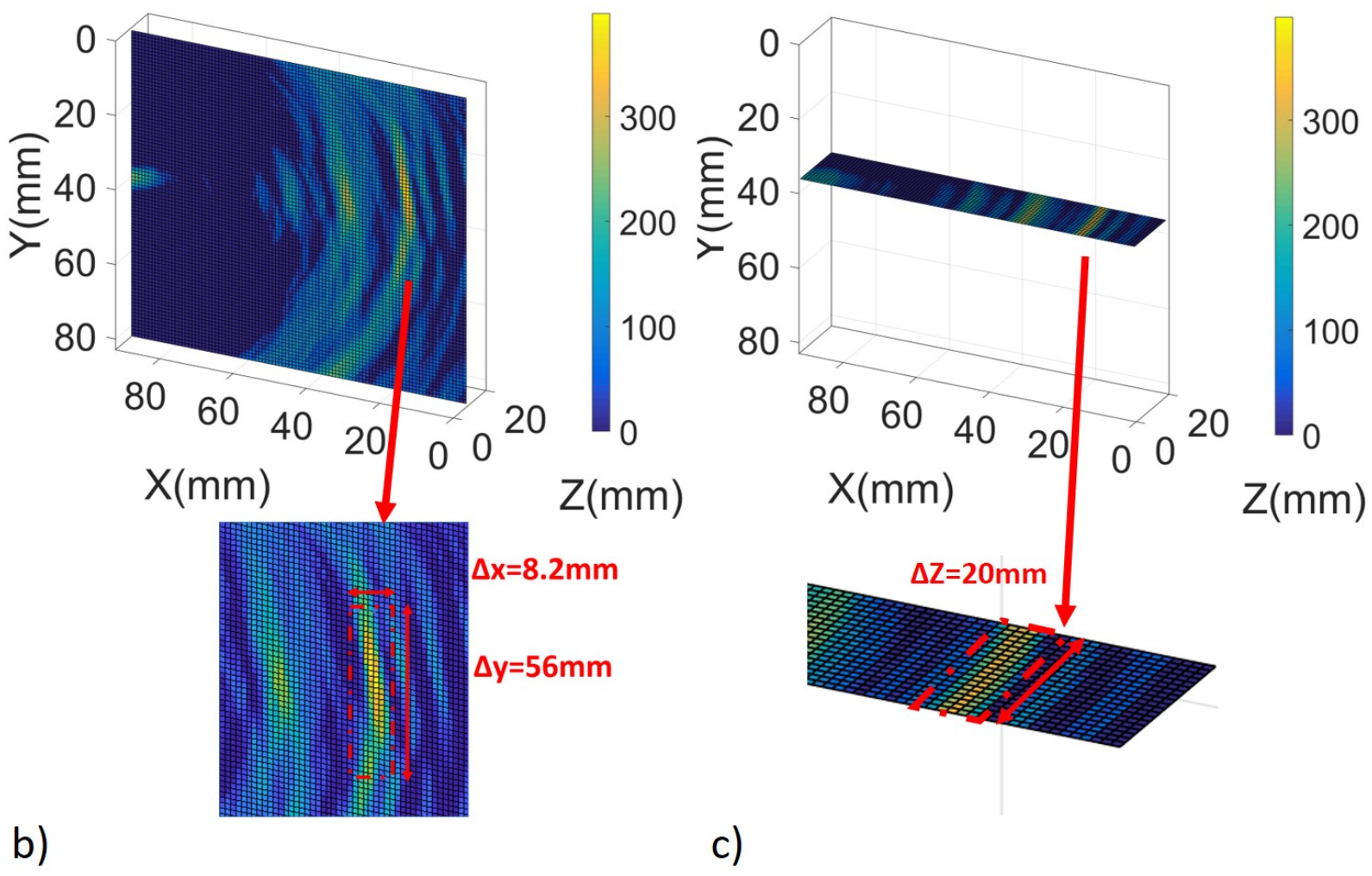

Figure 5: Reconstruction results of SAFT technique for a) 3D volume representation b) 3D slice for XY plane c) 3D slice for XZ plane. 


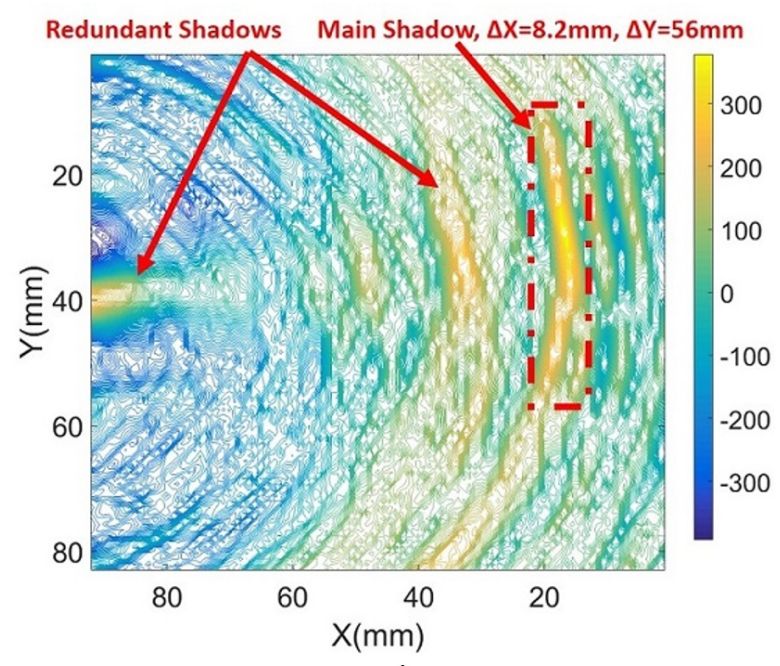

a) of the main shadow.

Using our actual experimental setup, SAFT algorithm and post-processing, these shadows cannot be directly removed. However, it is important to remove those redundant shadows for better visualization to avoid confusion between main echo and other echoes and to distinguish which of the shadows is the one we are interested in. We consider the main shadow is the one with higher amplitude and the redundant shadows are rather faded away with low amplitudes. We added an image processing filter To remove the redundant shadows. We applied an amplitude threshold filter that removes any point in the reconstructed image with amplitude less than $65 \%$ of the maximum amplitude. This threshold value was selected after visualizing the unfiltered reconstructed images. We found that the points with high intensity are starting around color map of $65 \%$. This leaves only the main shadow as the reconstruction of the defect with minor traces of the other shadows that are negligible. The results in Figure $6 \mathrm{~b}$ represent the contour map representation of Figure $6 \mathrm{a}$ after adding the rejection threshold of $65 \%$. A cylinder representing the actual defect shape is superimposed on the reconstructed images to represent the correlation between the main shadow location and the actual defect location. It should be noted that adding this rejection threshold filter is also removing some important data from the main shadow, hence it is important to calculate the size errors based on the unfiltered reconstructed image of Figure 6a. Fine tuning was applied to this filter to get optimum results. We can add a more restrictive filter to remove completely those negligible minor traces of redundant shadows ,but this would affect the size of the main shadow and would result in losing important data. Thus, there is a compromise between neglecting the redundant shadows and losing some intensity data

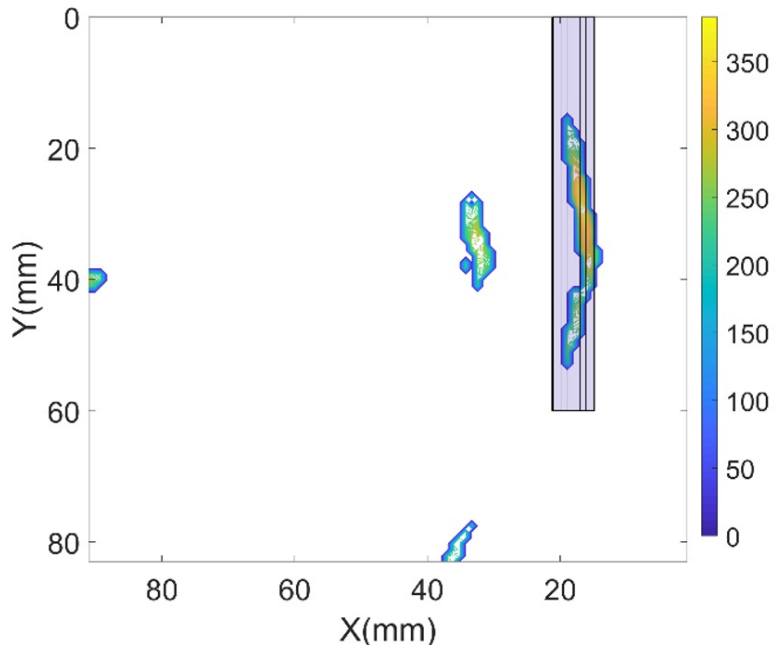

b)
Figure 6: a) Contour map of the defect at XY plane at $\mathrm{Z}=10 \mathrm{~mm}$ using SAFT technique: b) contour map of Figure 6 a after applying a reject threshold for data with an intensity below $65 \%$ with original geometry of the defect superimposed.

Comparing the results presented in this section with the previous B-scan ones, it should be noted that B-scan measurements allow the defect reconstruction only from one per- 
spective, and with a limited resolution. The implementation of the 2D scanning area and the signal processing using the SAFT technique over the object's volume allows the defect visualization from different angles and at different depths, making possible a 3D image reconstruction. For this application laser generated ultrasound excitation is much easier to implement than scanning with conventional transducers as it can be applied and scanned remotely and therefore the volume in which the ultrasound is generated is smaller. In the experiment presented here we scanned the laser across MxN points, and we used only two fixed ultrasonic receivers. This helped make the scan easier and show that by increasing the number of exciters (laser scan points in our case), we can obtain the same good-quality images with a very limited number of receivers fixed at particular points.

SAFT technique is sensitive to defects in agreement with the size of the measuring transducers. It is able to detect and reconstruct 3D defects at relatively large angles despite the corresponding errors. The position of the sensor and scan points do not need strict rules as long as the angle is within the allowable limits and the scan points as well as the sensors are at a relatively far distance from the boundaries of the object. The resolution of the reconstruction is in a range between those of the classical ultrasonic and the full optical methods. The algorithm is having a very high signal-to-noise ratio of the defects relative to other techniques.

\section{Conclusion}

We applied and compared two different techniques for defect localization and reconstruction, both using a hybrid system composed by laser generated ultrasound and conventional transducer receivers. The results obtained with both methods, the B-scan and the SAFT technique, indicate similar position of the defect, confirming the reliability of both algorithms. The SAFT technique offers the advantage of visualizing the defect in $3 \mathrm{D}$ and obtaining the projections of the sample planes, making it easier to distinguish the location of the defect in a 3D view, instead of just getting the one dimensional or two-dimensional results commonly used. We used the discussed algorithms to localize the defect position with a good signal-to-noise ratio, taking into consideration the limitations of structural dimensions. We believe that this hybrid approach combines different advantages of both photonic and ultrasonic devices, reducing the drawbacks of both methods. The remote control of the broad band excitation, possibility of scanning large areas and reduced number of receivers make it easy to implement for different materials and applications.

\section{Acknowledgements}

The work was supported by Spanish Ministry of Economy and Innovation (MINECO) and European Union FEDER through project FIS2015-65998-C2-1 and FIS2015-65998-C22 and by project AICO/2016/060 by Consellería de Educación, Investigación, Cultura y Deporte de la Generalitat Valenciana. 


\section{References}

[1] S. C. Her, S. T. Lin, Non-destructive evaluation of depth of surface cracks using ultrasonic frequency analysis, Sensors (Switzerland) 14 (9) (2014) 17146-17158. doi:10.3390/s140917146.

[2] B. Mi, J. E. Michaels, T. E. Michaels, An ultrasonic method for dynamic monitoring of fatigue crack initiation and growth, The Journal of the Acoustical Society of America 119 (1) (2006) 7485. doi:10.1121/1.2139647.

URL http://asa.scitation.org/doi/10.1121/1.2139647

[3] S. Ham, H. Song, M. L. Oelze, J. S. Popovics, A contactless ultrasonic surface wave approach to characterize distributed cracking damage in concrete, Ultrasonics 75 (2017) 46-57. doi:10.1016/j.ultras.2016.11.003.

[4] U. Amjad, S. K. Yadav, T. Kundu, Detection and quantification of pipe damage from change in time of flight and phase, Ultrasonics 62 (2015) 223-236. doi:10.1016/j.ultras.2015.05.022.

[5] M. Kharrat, L. Gaillet, Non-destructive evaluation of anchorage zones by ultrasonics techniques, Ultrasonics 61 (2015) 52-61. doi:10.1016/j.ultras.2015.03.007.

[6] B. Masserey, C. Raemy, P. Fromme, High-frequency guided ultrasonic waves for hidden defect detection in multi-layered aircraft structures, in: Ultrasonics, Vol. 54, 2014, pp. 1720-1728. doi:10.1016/j.ultras.2014.04.023.

[7] S. Delrue, K. Van Den Abeele, E. Blomme, J. Deveugele, P. Lust, O. B. Matar, Two-dimensional simulation of the single-sided air-coupled ultrasonic pitch-catch technique for non-destructive testing, Ultrasonics 50 (2) (2010) 188-196. doi:10.1016/j.ultras.2009.08.005.

[8] S. Delrue, M. Tabatabaeipour, J. Hettler, K. Van Den Abeele, Applying a nonlinear, pitch-catch, ultrasonic technique for the detection of kissing bonds in friction stir welds, Ultrasonics 68 (2016) 71-79. doi:10.1016/j.ultras.2016.02.012.

[9] T. Kreis, Application of Digital Holography for Nondestructive Testing and Metrology: A Review, IEEE Transactions on Industrial Informatics 12 (1) (2016) 240-247. doi:10.1109/TII.2015.2482900.

[10] K. Zhang, Z. Zhou, J. Zhou, Application of laser ultrasonic method for on-line monitoring of friction stir spot welding process, Appl. Opt. 54 (25) (2015) 7483-7489. doi:10.1364/AO.54.007483. URL http://ao.osa.org/abstract.cfm?URI=ao-54-25-7483

[11] Y. K. Zhu, G. Y. Tian, R. S. Lu, H. Zhang, A review of optical NDT technologies, Sensors 11 (8) (2011) 7773-7798. doi:10.3390/s110807773.

[12] S. Boonsang, J. Zainal, R. J. Dewhurst, Synthetic aperture focusing techniques in time and frequency domains for photoacoustic imaging, Insight: Non-Destructive Testing and Condition Monitoring 46 (4) (2004) 196-199. doi:10.1784/insi.46.4.196.55648.

[13] M. Spies, H. Rieder, Synthetic aperture focusing of ultrasonic inspection data to enhance the probability of detection of defects in strongly attenuating materials, NDT and E International 43 (5) (2010) 425431. doi:10.1016/j.ndteint.2010.04.002.

URL http://dx.doi.org/10.1016/j.ndteint.2010.04.002

[14] A. Ganguli, C. M. Rappaport, D. Abramo, S. Wadia-Fascetti, Synthetic aperture imaging for flaw detection in a concrete medium, NDT and E International 45 (1) (2012) 79-90. doi:10.1016/j.ndteint.2011.09.004. URL http://dx.doi.org/10.1016/j.ndteint.2011.09.004

[15] A. N. Sinclair, J. Fortin, B. Shakibi, F. Honarvar, M. Jastrzebski, M. D. C. Moles, Enhancement of ultrasonic images for sizing of defects by time-of-flight diffraction, NDT and E International 43 (3) (2010) 258-264. doi:10.1016/j.ndteint.2009.12.003. URL http://dx.doi.org/10.1016/j.ndteint.2009.12.003

[16] J. A. Jensen, S. I. Nikolov, K. L. Gammelmark, M. H. Pedersen, Synthetic aperture ultrasound imaging, Ultrasonics 44 (SUPPL.). doi:10.1016/j.ultras.2006.07.017.

[17] M. Spies, H. Rieder, A. Dillhöfer, V. Schmitz, W. Müller, Synthetic aperture focusing and time-of-flight diffraction ultrasonic imaging - Past and present, in: Journal of Nondestructive Evaluation, Vol. 31, 2012, pp. 310-323. doi:10.1007/s10921-012-0150-z.

[18] T. Stepinski, F. Lingvall, Synthetic aperture focusing techniques for ultrasonic imaging of solid 
objects, Synthetic Aperture Radar (EUSAR), 2010 8th European Conference on (2010) 14doi:papers2://publication/uuid/72BB2E26-227F-4027-9433-3990165E5916.

URL http://ieeexplore.ieee.org/xpls/abs $\left\{\backslash_{-}\right\}$all.jsp?arnumber $=5758760$ 